\title{
RESPONSABILIDAD POR NEGLIGENCIA MÉDICA Y ADMINISTRATIVA EN LA PRÁCTICA DEL PROCEDIMIENTO DE INTERRUPCIÓN VOLUNTARIA DEL EMBARAZO "IVE" EN LOS CASOS AUTORIZADOS EN COLOMBIA
}

\author{
Lizzeth Tatiana Aguillón Gómez* \\ Ruth Ardila Niño" \\ Ángela María Tavera Patiño* \\ Recibido: Enero 22 de 2016 \\ Aprobado: Mayo 20 de 2016
}

\section{RESUMEN}

Los objetivos específicos consisten en analizar el derecho constitucional a la IVE a partir de la noción de los derechos sexuales y reproductivos, estudiar el alcance del derecho a la IVE en Colombia con base a las subreglas jurisprudenciales establecidas por la Corte Constitucional, identificar los escenarios y las personas (naturales o jurídicas) a las que se les pueda atribuir responsabilidad por la dilación en la práctica de la IVE y las consecuencias jurídicas que se derivan de dicha responsabilidad y proponer recomendaciones en materia de atención de solicitudes tendentes a interrumpir un embarazo con el fin de evitar las dilaciones injustificadas por parte de las entidades prestadoras de los servicios de salud para garantizar el derecho de las mujeres a la IVE.

Se trata de una investigación exploratoria y descriptiva por cuanto, lo primero, se efectúa sobre un tema que no ha sido estudiado por nuestra legislación, por lo que sus resultados constituyen una visión aproximada de dicho tema, es decir, un nivel superficial de conocimiento. Y lo segundo, por cuanto se describe una necesidad en la sociedad de elaborar legislación sobre el tipo de responsabilidad que debe recaer en las EPS o IPS por demorar la práctica de la intervención voluntaria del embarazo.

\footnotetext{
* Abogada, Especialista en Responsabilidad Médica, Universidad Autónoma de Bucaramanga. Correo electrónico: tatianaaguillon@hotmail.com

** Abogada, Especialista en Responsabilidad Médica, Universidad Autónoma de Bucaramanga. Correo electrónico: ruthardilanino@gmail.com

*** Abogada, Especialista en Responsabilidad Médica, Universidad Autónoma de Bucaramanga. Correo electrónico: tavera85@hotmail.com
} 
Se concluyó que las demoras en la autorización e inclusive, en la realización del procedimiento, pueden causar daño psicológico en las mujeres que toman la decisión de interrumpir su embarazo y que la legislación colombiana no ha tocado el tema de la responsabilidad de tipo administrativo que debe recaer sobre el personal médico llamado a practicar la IVE o la misma entidad prestadora del servicio de salud. En ese sentido, es indispensable establecer pautas para crear un régimen de sanciones para quienes coloquen barreras administrativas que impidan el acceso a este servicio a las mujeres que por una buena razón se ven en la necesidad de suspender su embarazo.

Palabras clave: Responsabilidad, IVE, EPS, daño, IPS.

\section{RESPONSIBILITY FOR MEDICAL AND ADMINISTRATIVE NEGLIGENCE IN THE PRACTICE OF THE PROCEDURE OF VOLUNTARY INTERRUPTION OF THE PREGNANCY "IVE" IN THE CASES AUTHORIZED IN COLOMBIA} ABSTRACT

This article analyzes the constitutional right to the IVE from the notion of sexual and reproductive rights. Study the range of the right to IVE in Colombia, based on jurisprudential sub-rules established by the Constitutional Court. Also, identify the stage and persons (natural or legal) to which can be attributed to them responsibility for the delay in the practice of the IVE and the legal consequences of that responsibility. Finally, propose recommendations as for attention of request designed to terminate a pregnancy in order to avoid unjustified delays by the entities providing health services to ensure the right of women to the IVE.

This is an exploratory and descriptive research for two reasons. First, research approaches a topic that has not been studied by our legislation, for what their results constitute an approximate vision of the mentioned topic, a superficial level of knowledge. Second, it describes a need in society described to develop legislation on the type of responsibility that must relapse into the EPS or IPS for delaying the practice of voluntary intervention of pregnancy.

It was concluded that the delay in the authorization and inclusive, in the realization of the procedure, they could cause psychological damage to women, who decide to terminate their pregnancy and that Colombian legislation has not touched the issue of responsibility of administrative type that must relapse on the medical personnel called to practice the IVE or the entity providing the health service. 
Keywords: responsibility, IVE, EPS, damage, IPS.

\section{RESPONSABILIDADE POR NEGLIGÊNCIA MÉDICA E ADMINISTRATIVA NA PRÁTICA DO PROCEDIMENTO DE INTERRUPÇÃO VOLUNTÁRIA DA GRAVIDEZ "IVG" NOS CASOS PERMITIDOS NA COLÔMBIA RESUMO}

Os objetivos específicos consistem em analisar o direito constitucional à IVG a partir da noção dos direitos sexuais e reprodutivos, estudar a extensão do direito à IVG na Colômbia baseada nas sub-regras jurisprudenciais estabelecidas pela Corte Constitucional, identificar os cenários e pessoas (natural ou jurídica) às quais pode ser atribuída a responsabilidade pelo atraso na prática da IVG e as consequências jurídicas que se derivam dessa responsabilidade e propor recomendações na matéria de atenção de solicitudes destinadas a interromper a gravidez com o objetivo de evitar o atraso não justificado pelas entidades que prestam serviços de saúde para garantir o direito das mulheres a IVG.

Esta é uma pesquisa exploratória e descritiva, em que, no primeiro lugar, realiza-se sobre um assunto que não tem sido estudado por nossa legislação, os resultados constituem uma visão aproximada do tópico, ou seja, um nível superficial do conhecimento. No segundo lugar, por quanto se descreve uma necessidade na sociedade de elaborar legislação sobre o tipo de responsabilidade que deve recair sobre as EPS ou IPS por atrasar a prática da interrupção voluntária da gravidez.

Concluiu-se que os atrasos na autorização e até mesmo, na realização do procedimento pode causar danos psicológicos para as mulheres que decidem interromper a gravidez e que a legislação colombiana não tenha abordado a questão da responsabilidade administrativa que deve recair sobre o pessoal médico chamado a praticar a IVG ou a mesma entidade prestadora do serviço de saúde. Nesse sentido, é essencial estabelecer diretrizes para a criação de um regime de sanções para aqueles que coloquem barreiras administrativas que impedem o acesso a este serviço às mulheres que por boas razões são têm a necessidade de suspender sua gravidez.

Palavras chaves: Responsabilidade, IVG, Entidades Prestadoras de Serviços, danos, Instituições Prestadoras de Serviços 


\section{INTRODUCCIÓN}

El problema jurídico consiste en establecer si: ¿La dilación injustificada por parte de las EPS, las Instituciones Prestadoras de los servicios médico asistenciales y los profesionales de la salud, al no autorizar dentro de un término prudente el procedimiento requerido para la Interrupción Voluntaria del Embarazo, genera responsabilidad por los daños morales y fisiológicos causados a la madre que solicita la IVE?

Hoy día en Colombia se han presentado inconvenientes para la protección efectiva de los derechos fundamentales a la salud y vida de las personas, sin importar a qué régimen de seguridad social en salud pertenecen. Entre esas vulneraciones que ponen en riesgo estos derechos se encuentran las barreras administrativas impuestas de forma injustificada por parte de las instituciones prestadoras del servicio de salud - IPS-, entidades prestadoras del servicio de salud - EPS-, centros médicos, hospitales, clínicas, etc., ya sean privadas o públicas, para la realización de procedimientos médicos que se encuentran reglamentados en el ordenamiento jurídico colombiano, como la Interrupción Voluntaria del Embarazo - IVE-, a partir de los casos contemplados en la Sentencia C-355 de 2006 de la Corte Constitucional.

El Estado debe evitar que se ponga en riesgo y se amenace la vida y salud de las mujeres embarazadas que solicitan este procedimiento en atención a los requisitos que fueron establecidos en la referida sentencia. La solicitud de la IVE se realiza ante la EPS o EPS-S respectiva, la cual debe autorizar su práctica en el menor tiempo posible, pero en algunos casos por razones injustificadas, se exigen más requisitos de los que la sentencia y su posterior desarrollo jurisprudencial establecen, o demoran la autorización, obligando a las usuarias a acudir a la acción de tutela para que se realice dicho procedimiento.

Estos retrasos generan potenciales daños morales y secuelas sicológicas en las madres gestantes, quienes tienen que llevar el embarazo y crecimiento del feto sin quererlo, así como los posibles daños o secuelas físicas al bebé prematuro o en el peor de los escenarios, que muera unas semanas después de haber nacido. El artículo busca establecer si dichos daños generan responsabilidad tanto para la EPS, o IPS, e inclusive, para el personal médico que se niega a su práctica.

El marco teórico se desarrolla teniendo en cuenta conceptos como el derecho fundamental a la salud y a la vida en la ley colombiana, la 
intervención voluntaria del embarazo y las especificaciones contenidas en la sentencia C-355 de 2006; los derechos sexuales y reproductivos; las personas naturales y jurídicas encargadas de la prestacion del servicio de salud en Colombia (personal médico, EPS, IPS), los derechos y deberes de los pacientes, el concepto de daño, la distinción entre daño y perjuicio, los diferentes tipos de responsabilidad que pueden atribuírsele a estas entidades y personas, la objeción de conciencia que puede convertirse en una barrera importante para la demora en la realización del procedimiento, los riesgos para la mujer por la práctica a destiempo de la IVE y los protocolos con los que cuentan las EPS SURA MEDICINA PREPAGADA y CAFESALUD EPS para la práctica de la IVE.

El artículo lleva las connotaciones de una investigación exploratoria y descriptiva por cuanto, lo primero, se efectúa sobre un tema que no ha sido estudiado por nuestra legislación, por lo que sus resultados constituyen una visión aproximada de dicho tema; es decir, un nivel superficial de conocimiento. Y lo segundo, por cuanto se describe una necesidad en la sociedad de elaborar legislación sobre el tipo de responsabilidad que debe recaer en las EPS, IPS, y personal médico, por dilatar la práctica de la intervención voluntaria del embarazo. Para ello, el análisis a realizar acerca de los protocolos que se llevan a cabo para dicho procedimiento y donde se pueden señalar algunos obstáculos de tipo administrativo que puedan llevar a consecuencias no deseadas por parte de las madres gestantes.

Para la recolección de la información se acudirá a la ley, jurisprudencia y doctrina colombiana sobre el tema

Entonces, se pretende identificar los riesgos que pueden presentarse al no practicarse de manera perentoria la IVE cuando es solicitada por alguna mujer que se encuentre en cualquiera de los tres (3) requisitos que exige la Corte Constitucional para su práctica legal y establecer responsabilidades.

\section{DERECHO FUNDAMENTAL A LA VIDA Y LA SALUD EN EL ORDENAMIENTO JURÍDICO COLOMBIANO}

\subsection{Derecho fundamental a la vida}

El derecho a la vida es un derecho universal y fundamental que todo ser humano tiene, que debe respetarse por sobre toda circunstancia y que 
solo debe terminar por causas naturales y frente a este hay ligados otros derechos como la vida, la libertad de expresión y dignidad humana. Es un derecho que se encuentra plasmado en los ordenamientos jurídicos de todas las naciones y en la Costitución Política de Colombia está circunscrito en el artículo $11^{\circ}$ que dice “... El derecho a la vida es inviolable. No habrá pena de muerte", convirtiéndose así en una garantía constitucional absoluta.

La Carta reconoce que hay vida desde la fecundación del óvulo con el espermatozoide, por lo que se impone la protección de dicho derecho a partir de la concepción.

\subsection{EI derecho fundamental a la salud}

La salud ha sido definida por la ONU, a través de la Organización Mundial de la Salud (OMS) como "... un estado de completo bienestar físico, mental y social y no solamente la ausencia de afecciones o enfermedades (...) el goce del grado máximo de salud que se pueda lograr es uno de los derechos fundamentales de todo ser humano sin distinción de raza, religión, ideología política o condición económica o social (...) considerada como una condición fundamental para lograr la paz y la seguridad" (Constitución de la Organización Mundial de la Salud)

Por su parte, la Corte Constitucional colombiana ha dicho que,

(...) el derecho a la salud es un derecho fundamental de carácter autónomo. Según el artículo 49 de la Constitución Política, la salud tiene una doble connotación -derecho constitucional y servicio público-. En tal sentido, todas las personas deben poder acceder al servicio de salud y al Estado le corresponde organizar, dirigir, reglamentar y garantizar su prestación de conformidad con los principios de eficiencia, universalidad y solidaridad" (Corte Constitucionao, Sentencia T-737 de 2013)

La Ley 1751 de 16 de febrero de 2015 estatutaria de la salud, en su artículo $2^{\circ}$ define la naturaleza y el contenido del derecho fundamental a la salud en los siguientes términos:

El derecho fundamental a la salud es autónomo e irrenunciable en lo individual y en lo colectivo. Comprende el acceso a los servicios de salud de manera 
oportuna, eficaz y con calidad para la preservación, el mejoramiento y la promoción de la salud. El Estado adoptará políticas para asegurar la igualdad de trato y oportunidades en el acceso a las actividades de promoción, prevención, diagnóstico, tratamiento, rehabilitación y paliación para todas las personas....

También resalta los elementos propios de este derecho como la disponibilidad, aceptabilidad, accesibilidad, calidad e idoneidad del personal, universalidad, equidad, continuidad, oportunidad, prevalencia de derechos, libre elección, eficiancia, y proteccion a pueblos indígenas. (Ley 1751 de 16 de febrero de 2015 artículo 6)

\subsection{La interurpción voluntaria del embarazo (IVE) y la Sentencia C- 355 de 2006}

El aborto se define como la interrupción del embarazo antes de la semana número 23 de gestación y se puede producir de manera instantánea, ya sea por causas naturales o de manera inducida.

Existen diferentes posturas acerca de este tema, porque están comprometidos valores morales y éticos. Quizás la postura más radical frente a la IVE es la que tiene la Iglesia Católica, pues no está de acuerdo con su práctica, ni siquiera como forma terapéutica para salvaguardar la vida de la madre gestante.

De otra parte se encuentra el criterio médico, cuyo deber es propender por la salud y vida del paciente. Así lo expresa Merchan Price:

(...) Y como es obvio que no puede concebirse la salud sin la vida, la medicina busca siempre y en todo lugar y circunstancia preservar la vida del enfermo. En ese sentido, el médico solo es un subordinado de ese fin y nunca lo es, en primera instancia, de la ley ni del Estado, ni de ningún grupo social y ni tampoco de ninguna ideología o religión que no respete el valor biológico de la vida. (Merchan, 2012, p. 34)

En nuestro pais, estas posturas absolutistas frente al derecho a la vida han venido cambiando y prueba de ello, el pronunciamiento de la Corte Constituicional en sentencia de constitucionalidad C-355 de 2006 que despenalizó el aborto en tres eventos que serán objeto de estudio más adelante. 
La génesis de este fallo comienza en el año 2005 cuando fueron admitidas por la Corte Contitucional, demandas en contra de los artículos 122, 123, 124 y el numeral $7^{\circ}$ del artículo 32 del Código Penal (Ley 599 de 2000), pues se consideraba que las normas violaban derechos como la dignidad humana, la autonomia reproductiva, el libre desarrollo a la personalidad, la igualdad, la vida y la salud, entre otros derechos fundamentales.

En dicho pronunciamiento, se habló del aborto y el derecho comparado en Alemania y Europa Occidental frente al mismo, donde la legislación sobre el tema ha sido muy dispar y solo a partir de 1990 se empezó a considerar dentro de las legislaciones de diferentes naciones,la posibilidad de despenalizarlo ${ }^{1}$. La Corte Constitucional decidió lo que sigue, con salvamento de voto del H. M. Álvaro Tafur Galvis:

... Segundo. Declarar EXEQUIBLE el artículo 32, numeral 7 de la Ley 599 de 2000, por los cargos examinados en la presente sentencia.

Tercero. Declarar EXEQUIBLE el artículo 122 de la Ley 599 de 2000, en el entendido que no se incurre en delito de aborto, cuando con la voluntad de la mujer, la interrupción del embarazo se produzca en los siguientes casos: (i) Cuando la continuación del embarazo constituya peligro para la vida o la salud de la mujer, certificada por un médico; (ii) Cuando exista grave malformación del feto que haga inviable su vida, certificada por un médico; y, (iii) Cuando el embarazo sea el resultado de una conducta, debidamente denunciada, constitutiva de acceso carnal o acto sexual sin consentimiento, abusivo o de inseminación artificial o transferencia de óvulo fecundado no consentidas, o de incesto...".

En el pronunciamiento quedó claro que la Corte defiende el valor de la vida como fundamento ontológico del orden jurídico y el bien jurídico de la vida del nasciturus, pero dejó claro tambien, que respalda los derechos de la mujer por encima de aquel bien, constituyendo un bloque de contitucionalidad basado en la dignidad de la mujer, su derecho a la vida, a la igualdad, a la protección de su salud y su derecho a la libertad sexual y reproductiva, como titular de derechos que ella es, recibiendo mayor protección y peso, que el bien jurídico de la vida del que aun no es titutlar de ese derecho.

1 En América, por ejemplo, países como Argentina, Bolivia, Cuba y México permiten el aborto y en otros como Chile, Costa Rica, Ecuador, El Salvador, Guatemala y Honduras, el aborto está prohibido 


\subsection{Interrupción Voluntaria del Embarazo (IVE)}

Es un procedimiento mediante el cual se busca la interrupción del embarazo de manera consentida y voluntaria, sin que la mujer sea expuesta a obstáculos de otra clase, de manera insegura y poniendo en riesgo su vida. Voluntad que debe manifestarla al médico tratante de la IPS respectiva adjuntando los documentos requeridos, según el caso en que se encaje la circunstancia en la que es viable su práctica.

La Corte Constitucional a partir de la Sentencia C-355 de 2006 despenalizó el aborto en tres eventos y posteriormente, a través de sentencias, entre otras, la T-171 de 2007, T-988 de 2007, T-209 de 2008, T-009 de 2209, T585 de 2010 y T-841 de 2011, ratificó el derecho de las mujeres a decidir sobre la interrupción de su embarazo.

Las tres (3) circunstancias plasmadas en la referida sentencia son:

\begin{tabular}{|l|l|}
\hline \multicolumn{1}{|c|}{ CAUSAL } & \multicolumn{1}{|c|}{ REQUISITO } \\
\hline $\begin{array}{l}\text { 1. Cuando el embarazo constituya peligro para la salud o la } \\
\text { vida de la mujer. }\end{array}$ & Certificado de un (1) médico/a. \\
\hline $\begin{array}{l}\text { 2. Cuando exista grave malformación del feto que haga } \\
\text { inviable su vida. }\end{array}$ & Certificado de un (1) médico/a. \\
\hline $\begin{array}{l}\text { 3. Cuando el embarazo sea el resultado de una conducta } \\
\text { debidamente denunciada, constitutiva de acceso carnal o } \\
\text { acto sexual sin consentimiento (violación), abusivo, o de } \\
\text { inseminación artificial o de transferencia de óvulo } \\
\text { fecundado no consentida, o de incesto. }\end{array}$ & $\begin{array}{l}\text { Copia de la denuncia por } \\
\text { violación interpuesta ante } \\
\text { autoridad competente. }\end{array}$ \\
\hline
\end{tabular}

\subsection{Derechos sexuales y reproductivos}

Los derechos sexuales y reproductivos tienen su asidero jurídico en diferentes intrumentos internacionales (Declaración Universal de Derechos Humanos, Pacto Internacional de Derechos Civiles y Políticos, Pacto de Derechos Económicos y Sociales, Convención Internacional para la Eliminación de todas las Formas de Discriminación Contra la Mujer, entre otros), que hacen referencia a los derechos humanos, y se han catalogado como tales, por ejemplo, el derecho a la vida, a la integridad física, psíquica y social, libertad del ejercicio de la sexualidad, respecto a las decisiones personales sobre orientación sexual, respeto a la opción de reproducción, libertad de fundar una familia, libertad de decidir sobre el número de hijos, derecho a la igualdad de sexo y de género, libertad de elegir compañero sexual, expresión y libre ejercicio de la orientación sexual, el derecho a elegir si se tienen relaciones sexuales, derecho a la información científica acerca de la sexualidad y el derecho a la intimidad personal. (Ministerio de Educación, Colombia, s.f) 
Como componentes de la sexualidad se encuentran los siguientes:

- Reconocimiento de la dignidad. Toda persona es valiosa por el solo hecho de ser humano y deben emprenderse acciones para que este postulado sea una realidad ante la sociedad, la familia y con la pareja.

- Valoración de sí mismo. Es reconocerse como un ser valioso y único que merece ser respetado y valorado y de ser necesario, buscar ayuda para defender los propios derechos.

- Desarrollo del juicio moral. Se refiere a la toma de decisiones frente a la sexualidad pues esta depende de los principios éticos y morales de cada persona.

- Proyecto de vida. Es la toma de decisiones a partir de los proyectos que permitan el libre desarrollo de la personalidad.

- Libre desarrollo de la personalidad. Es el actuar libre dentro de la limitación que impone el derecho de los demás y el orden jurídico. Cuando dicha limitación va en contra de la dignidad de las personas se debe hacer uso de los mecanismos jurídicos para su restablecimiento. (Ministerio de Educación, Colombia, s.f)

\section{PERSONAS NATURALES Y JURÍDICAS ENCARGADAS DE LA PRESTACIÓN DEL SERVICIO DE SALUD EN COLOMBIA}

\subsection{Entidades Promotoras de Salud}

La Ley 100 de 1993, "Por medio de la cual se crea el Sistema de Seguridad Social Integral y se dictan otras disposiciones", que en el artículo 177 define las EPS como “... entidades responsables de la afiliación, y el registro de los afiliados y del recaudo de sus cotizaciones, por delegación del Fondo de Solidaridad y Garantia". Importa resaltar que, estas entidades no prestan servicios médicos asistenciales sino que comportan un esquema de aseguramiento dirigido a la población.

Su función básica es organizar, garantizar, directa o indirectamente, la prestación del plan de salud obligatorio (POS) a sus afiliados y girar la diferencia que existe entre los ingresos adquiridos por las cotizaciones 
de sus afiliados y el valor correspondiente en unidades de pago por capitación al Fondo de Solidaridad y Garantía (FOSYGA).

\subsection{Instituciones Prestadoras del Servicio}

Son instituciones privadas que prestan servicios médicos de consulta, hospitalarios, clínicas y cuidados intensivos a los usuarios. (Ej: centros de diagnósticos, consultorios médicos, clìnicas, centros de salud, laboratorios clínicos, etc.). No deben confundirse con las Empresas Sociales del Estado que son de carácter público.

\subsection{Profesionales de la Salud}

Es el personal humano (profesionales o auxiliares) que presta los servicios médicos a los afiliados al sistema general de seguridad social en salud que lo requieran, ya sean cotizantes o beneficiarios, en las diferentes áreas, como medicina, odontología, fonoaudiología, enfermería, sicología, bacteriología, fisioterapia, terapia ocupacional, nutrición, radiología y demás profesiones autorizadas por el Ministerio de la Salud.

La prestación de los servicios de estos profesionales son contratados por las EPS o IPS para poder atender y prestar el servicio a los afiliados.

La Ley 1751 de 2015 destacó un capítulo refiriéndose a dichos profesionales en cuanto a su autonomía, en los siguientes términos:

Artículo 17. Autonomía profesional. Se garantiza la autonomía de los profesionales de la salud para adoptar decisiones sobre el diagnóstico y tratamiento de los pacientes que tienen a su cargo. Esta autonomía será ejercida en el marco de esquemas de autorregulación, la ética, la racionalidad y la evidencia científica. Se prohíbe todo constreñimiento, presión o restricción del ejercicio profesional que atente contra la autonomía de los profesionales de la salud, así como cualquier abuso en el ejercicio profesional que atente contra la seguridad del paciente. La vulneración de esta disposición será sancionada por los tribunales $\mathrm{u}$, organismos profesionales competentes y por los organismos de inspección, vigilancia y control en el ámbito de sus... 


\subsection{Derechos y deberes de los pacientes}

Existe un catálogo extenso sobre los derechos de los pacientes que comienza por el derecho a la vida, a la salud y a la dignidad humana. Derechos que se generan a partir de la enfermedad que afecta a la persona y entre ellos podemos mencionar los siguientes: derecho a la integridad psicofísica, a la asistencia sanitaria, a disponer del propio cuerpo, a la intimidad, confidencialidad, privacidad, secreto médico, igualdad, a la verdad, a ser diferente, a ser tratado por un médico bondadoso, responsable, idóneo y prudente; el derecho a una muerte digna, a recibir o rechazar la asistencia espiritual o moral, a la atención médica de buena calidad, a la libertad de elegir o cambiar libremente de médico y hospital o institución de servicio de salud, derecho a la segunda opinión, y el derecho a la información. (Garay, 2009, p. 358-360)

Ahora bien, entre los deberes que les asisten a los pacientes están el de cuidar y velar por su salud, atender las recomendaciones que su médico tratante les indique, colaborar con información cierta que deban suministrar al profesional de la salud para obtener un acertado diagnóstico, y cumplir con las normas que la institución médica cuenta.

\section{EL DAÑO}

El profesor Bémoit afirma que:

(...) el daño es un hecho; es toda afrenta a la integridad de una cosa, de una persona, de una actividad, o de una situación (...) el perjuicio lo constituye el conjunto de elementos que aparecen como las diversas consecuencias que se derivan del daño para la víctima del mismo. Mientras que el daño es un hecho que se constata, el perjuicio es, al contrario, una noción subjetiva apreciada en relación con una persona determinada. (Bénoit. 1975, I, p. 1351)

Para el escenario que se aborda en este artículo, el daño que puede producirse en una mujer que solicita la IVE y no se le practica o su realización es demorada, tiene varias aristas, pues no solo puede constituirse en un daño fisiológico en el aparato reproductor de la gestante, con consecuencias indeseables para la paciente, sino que también, en lo emocional, que puede darse en cualquiera de los tres eventos de despenalización del aborto. 
En el ámbito del perjuicio, las secuelas pueden ser graves y difíciles de superar, pues la mujer decide suspender su embarazo siempre por una buena razón, se insiste, más no por simple deseo o capricho. Consecuencias como la infertilidad por un procedimiento mal practicado, daño en su cuerpo o enfermedades colaterales que aparecen con ocasión de una demora o falla en el procedimiento, e inclusive, cuando se insiste en la no suspensión del embarazo por parte del personal médico, y las consecuencias de ordén psicológico y mental que pueden darse en los casos de violación o cuando el feto presenta malformaciones en su cuerpo y sin embargo la mujer está obligada a dar a luz a pesar de su decisión de no continuar con el embarazo, llevando a la aparición de la depresión, enfermedad mental de amplia presencia en el ordén mundial, e inclusive a patologías más extremas que afectan la salud mental de la mujer, y que pueden desarrollarse, son eventos de gran impacto que pueden presentarse en las pacientes ante la no práctica del procedimiento.

Estas lesiones implican un golpe en lo familiar y social que no hay que dejar a un lado, pues la mujer queda expuesta al escarnio público, muy a pesar de que el tratamiento se lleve bajo estricta reserva, en razón a la propia vivencia que experimenta la paciente por tomar una decisión que puede tener graves consecuencias para su salud tanto mental como fisiológica.

\section{EL CONCEPTO DE RESPONSABILIDAD APLICABLE A LOS EVENTOS DE INTERRUPCIÓN VOLUNTARIA DEL EMBARAZO}

\subsection{Responsabilidad}

Según el Diccionario de la Lengua Española, la responsabilidad es "una obligación que hay que cumplir" y más estrictamente, es "una obligación de reparar y satisfacer por sí o por otro, una deuda o un daño" (Pantoja, 2015, p. 64). Concepto que nos acerca de manera amplia al Derecho Civil, pues es una obligación de reparar.

Se entiende que la responsabilidad cabe dentro de un abanico particular de conceptos como la responsabilidad moral, penal, fiscal, disciplinaria, civil, (contractual o extracontractual), responsabilidad por culpa probada, por culpa presunta, responsabilidad de resultados, responsabilidad principal y subsidiaria. 
Puede ser directa (cuando el individuo comete un acto antijurídico y se le aplica una sanción), indirecta (cuando es susceptible de ser sancionado por culpa atribuible a un tercero), subjetiva (se requiere para la imposición de la sanción que la persona haya querido o previsto el resultado de su conducta antijurídica) y objetiva (aquella donde se sanciona independiente si el actor quiso o previó el acto antijurídico).

\subsection{Responsabilidad civil}

La responsabilidad civil o llamada también derecho de daños, es "la obligación de asumir todas las consecuencias patrimoniales $\mathrm{o}$ económicas que nacen de un acto, conducta o hecho, que ocasiona un daño a otro" (Pantoja, 2015, p. 83). Aquí el elemento esencial es el daño, pues nadie puede perjudicar a otro sin justificación alguna.

Tamayo Jaramillo (2010) define la responsabilidad civil como, “... la consecuencia jurídica en virtud de la cual quien se ha comportado en forma ilícita, debe indemnizar los daños, producidos a terceros...", encontrando la responsabilidad civil, como fuente de obligaciones, en los hechos ilícitos.

Para el Derecho Civil la responsabilidad se entiende como la obligación de indemnizar el daño causado, por lo que su origen se encuentra en los preceptos que regulan las fuentes de las obligaciones.

Ahora bien, según el Código Civil colombiano la responsabilidad civil puede ser, como se dijo anteriormente, contractual o extracontractual:

\subsubsection{Responsabilidad contractual}

Tiene su comienzo en el daño que surge por el incumplimiento de las obligaciones contractuales. Pablo Rodríguez la define como la obligación de indemnizar los perjuicios causados por el incumplimiento de una obligación preexistente de una relación contractual $(2009$, p. 9)

Descendiendo al caso de la responsabilidad médica, puede ser que en la relación entre el médico y el paciente exista un acuerdo de voluntades (expreso o tácito); concretándose un vínculo contractual. No obstante, puede haber ausencia de voluntad de una de las partes, que impide la existencia de una relación contractual. En este último escenario nacería una responsabilidad extracontractual. Sin embargo, luego de múltiples debates referidos a definir si la responsabilidad derivada del servicio 
médico es de una $u$ otra naturaleza, se ha concretado que la responsabilidad galénica es contractual y excepcionalmente lo es extracontractual.

Es contractual por cuanto el contrato médico mantiene sus elementos:

a) Civil. El contrato es civil, sujeto al Código Civil y al fuero respectivo pues el origen liberal de la profesión médica lo hace ajeno al derecho mercantil.

b) Intuite personae. Con las limitaciones que presenta la prestación del servicio de salud desde los años 80 (a raíz de la aparición de la medicina prepagada), la relación entre las partes es de confianza. El profesional también puede elegir al paciente, o puede negarse a prestar la atención médica por motivos de especialidad médica o personales.

c) Bilateral. Origina obligaciones y responsabilidades para el médico de diligencia y prudencia en el cumplimiento de sus deberes de recuperación, conservación y preservación de la salud de las personas y para el paciente, los deberes de informar, colaborar, cumplir con el manejo terapéutico, pagar los honorarios, etc.

d) Oneroso. El paciente se obliga a abonar la prestación del servicio de salud brindada por el médico. En caso de pactarse la gratuidad del servicio, ello no libera al galeno de responsabilidad.

e) Conmutativo. Las prestaciones que obligan a cada parte a actuar de cierta forma son ciertas y se encuentran preestablecidas.

f) De tracto sucesivo. El contrato queda perfeccionado desde que las partes recíprocamente hubieran manifestado su consentimiento (expreso verbal o excepcionalmente escrito- o tácito) ya que por lo general, el paciente es parte del diagnóstico o tratamiento médico, el cual no se consuma de manera instantánea.

g) No formal. El contrato médico no está sujeto a forma determinada; es libre. Excepcionalmente se requiere de una forma determinada, como para los casos de trasplante de órganos.

h) Obligaciones principales y secundarias. El médico tiene una obligación principal de disponer los medios necesarios y adecuados 
para la cura del paciente y deberes secundarios de conducta como la de efectuar un correcto diagnóstico, brindar información verbal al paciente, contar con el consentimiento informado, prescribir, guardar el secreto médico, satisfacer el plan de prestación del servicio médico en su integridad, etc. (Caray, 2009, p. 532)

\subsubsection{Responsabilidad extracontractual}

Los supuestos de responsabilidad extracontractual pueden enmarcarse de la siguiente manera:

a) Atención médica a requerimiento de un tercero, sin que medie la voluntad del paciente; b) atención médica espontánea, cuando el servicio es prestado por el médico directamente sin intervención alguna de la voluntad del paciente (ej. médico que auxilia a la víctima en una vía pública) siempre y cuando esa atención medica no haya sido ratificada por el paciente asistido; c) cuando el actuar médico se configura en un delito; d) cuando el contrato celebrado entre médico y paciente es nulo por carecer de alguno de sus elementos o por ser ilícita la causa; e) en los eventos de atención a un incapaz, cuando no se pueda comunicar con el respectivo representante legal; f) cuando la actividad del médico es desarrollada en contra de la voluntad del paciente, en caso de tentativa de suicidio; g) actuación médica por imperativo legal, esto es, cuando la relación medico paciente es impuesta coactivamente a este, por una imposición legal o administrativa (en casos de urgencia o gravedad); h) cuando el actuar médico se hace con intención de causar daño, (dolo); i) cuando la actuación causa daño a terceros y/o extraños a la relación convencional existente con su paciente; j) el reclamo judicial de pacientes, en la hipótesis en que el paciente fallece como consecuencia de la atención médica y quienes reclaman son sus familiares, y, k) por omisión de auxilio.

\subsection{Responsabilidad administrativa}

Las actuaciones prohibidas ante la solicitud de la IVE han sido determinadas por la Superintendencia de Salud en la Circular 003 de 26 de abril de 2013 de la siguiente manera:

a) Realizar juntas médicas, de revisión o aprobación por auditores que ocasionan tiempos de espera injustificados para la práctica de la IVE.

b) Exigir a las adolescentes menores de 14 años embarazadas, el consentimiento de sus representantes legales para acceder al servicio. 
c) Exigir, dictámenes de medicina forense, órdenes judiciales, exámenes adicionales de salud, autorización de familiares, asesores jurídicos, auditores médicos, etc,, alegar objeción de conciencia colectiva e institucional, suscribir pactos individuales o conjuntos para negarse a la práctica de la IVE, acogerse a formatos de adhesión que incidan en que las entidades hospitalarias no cuenten en su planta de personal con médicos que presten el servicio, desconocer conceptos expedidos por sicólogos a quienes la Ley 1090 de 2006 les reconoce el estatus de profesionales de la salud y cualquier otra medida que conduzca al mismo resultado inconstitucional, pues constituye violación al régimen jurídico.

Ahora bien, en ese sentido se hace necesario describir otros escenarios posibles en donde se puede incurrir en prácticas dilatorias por parte de las entidades prestadoras del servicio médico, como por ejemplo, la demora en la asignación de citas a las pacientes que necesitan practicarse la IVE, la reprogramación de esas citas con la excusa de la famosa frase "no hay agenda", tan siquiera para el primer encuentro con el médico tratante de la paciente; la exigencia de otros requisitos que no están contenidos en las circulares y normas que regulan este procedimiento, la falta de profesionales en los centros de salud que no tienen obstáculos para practicar la IVE, como lo es la objeción de conciencia, la excusa de la falta de convenio con las IPS, el lleno de formularios innecesarios y conceptos médicos tardíos, el personal de salud que trata de convencer a las pacientes para que no se realicen el procedimiento ante justificaciones de índole religioso, moral o ético, y la exigencia de copagos o cuotas moderadoras cuando la mujer no cuenta con recursos económicos y pertenece al régimen subsidiado, entre otros.

Se cuestiona entonces, el tipo de responsabilidad que recae sobre estas instituciones y profesionales de la salud por la demora en autorizaciones y demás trámites que se convierten en barreras de acceso para las madres gestantes que optan por la IVE, con justificaciones válidas.

\section{LA OBJECIÓN DE CONCIENCIA}

Es un conflicto entre el derecho a la libertad de conciencia y el derecho a la IVE dentro de las causales de excepción. No obstante, la Corte Constitucional reconoce que este no es un derecho absoluto y su límite es la Constitución. El médico puede objetar conciencia por sus creencias y convicciones personales o por condiciones de tipo religioso o moral y nunca basarse en su opinión acerca de que si está de acuerdo o no con las razones 
por las que una mujer decide interrumpir su embarazo, garantizando siempre la satisfacción del derecho de la mujer a interrumpir el mismo.

La alta corporación en varios pronunciamientos ha establecido unos lineamientos para proteger estos dos derechos encontrados:

a. En situaciones de urgencia, en las que existe peligro inminente para la vida de la mujer y solo hay un prestador del servicio, no se puede alegar objeción de conciencia, y se debe prestar el servicio en cumplimiento de la obligación última de proteger os derechos fundamentales de la mujer. b. La objeción de conciencia solo aplica para el/la médico/a que realiza de manera directa el procedimiento, es decir, no puede ser ejercida por los otros profesionales que intervienen en el proceso (por ejemplo, personal de anestesiología, enfermería, orientación y asesoría, administradores hospitalarios, secretarias, jueces, etc.) ni para abstenerse de dar información, ni para coartar la voluntad de la mujer, ni persuadirla para cambiar su decisión. c. Implica la obligación de remitir a la mujer a otro profesional que no sea objetor de conciencia...". (Corte Constitucional, Sentencia T-209 de 2008)

Ahora, la Circular N. 003 de 26 de abril de 2013 emanada de la Superintendencia de Salud señala unos requisitos para ejercer el derecho a la objeción de conciencia por parte del personal médico: a) Debe presentarse por escrito y de manera individual donde se expongan los fundamentos que impiden al médico ejercer la práctica del procedimiento; b) No puede ser colectiva ni institucional, ni vulnerar los derechos fundamentales de las mujeres; c) Quien la alegue debe remitir de inmediato a la mujer a otro médico que esté en condiciones de realizar el procedimiento; d) Los prestadores del servicio de salud deben definir previamente los profesionales médicos que realicen el procedimiento y deben establecer mecanismos para determinar si la objeción de conciencia es o no procedente conforme a los parámetros establecidos por la profesión médica y demás normas.

\section{RIESGOS PARA LA MUJER POR LA NO PRÁCTICA DE LA IVE}

La Organización Mundial de la Salud (OMS) recomienda que todos los centros de atención en salud (niveles 1, 2 y 3 ) deben estar disponibles 
para prestar la IVE capacitando al personal médico tanto para proveer los servicios como para hacer las remisiones pertinentes.

Los centros de atención primaria deben tener la capacidad de ofrecer dichos servicios en los casos por aspiración endouterina hasta las 15 semanas y con medicamentos hasta la semana 10 de embarazo, en razón a que ambos son procedimientos ambulatorios.

Los centros de segundo nivel de atención, deben ofrecer todos los servicios y estar preparados para atender a la mujer en todas las etapas y circunstancias del embarazo. Sin embargo, deben estar disponibles para hospitalización en caso de presentarse algún riesgo para la paciente y deben estar capacitados para manejar las posibles complicaciones.

A los centros de atención de tercer nivel deben llegar los casos de mujeres que padezcan enfermedades coexistentes que requieran un trato especial de alta complejidad, brindando todo el apoyo necesario por las complicaciones en el manejo de la IVE. Por lo tanto deben contar con todo el personal idóneo y la capacidad física para realizar la IVE.

En la sentencia T-841 de 2011 la Corte Constitucional habló sobre los diferentes riesgos que pueden presentarse si la IVE que solicita una mujer no es practicada de manera perentoria y dentro de los términos establecidos por la misma Corte. Destacó para cada caso los conflictos o peligros que puede padecer una mujer si se le cercena dicho pedimento. Resalta la importancia del derecho al diagnóstico como un derecho ligado al de la salud, para poder acelerar y no dilatar todo el procedimiento que comienza precisamente con la consulta médica, en el entendido en que debe ser rápido y oportuno para evitar riesgos tanto físicos como sicológicos. De ahí la importancia de una correcta valoración médica para atender, remitir y practicar el procedimiento sin dar tiempo para secuelas emocionales que pueden quedar por el impacto que genera cualquiera de los tres (3) eventos despenalizados.

\section{PROTOCOLOS DE SALUD PARA LA PRÁCTICA DE LAIVE}

La Circular N. 003 del 25 de abril de 2013 contiene las instrucciones que deben seguir los prestadores de servicios de salud, las entidades administradoras de planes de beneficios, públicos o privados, y entidades territoriales, quienes están en la obligación de prestar el servicios de IVE a mujeres que se encuentren en cualquiera de los tres (3) escenarios establecidos en la sentencia C-355 de 2006, entre las que se 
exige que toda EPS y la red pública de salud deben garantizar el acceso de las mujeres a este servicio. Todas las EPS están en la obligación de acatar lo ordenado por la Corte Constitucional y realizar la IVE sin discriminar a quienes lo requieran, que el derecho a la objeción de conciencia no es institucional sino personal, y que el término razonable para practicar la IVE es de cinco (5) días contados a partir de la consulta.

Determinó igualmente que las EPS, hospitales, personal médico y administrativo deben abstenerse de realizar juicios de valor o de responsabilidad penal sobre las mujeres afiliadas a la entidad que solicite el procedimiento. Que sus actuaciones "se deberán dirigir exclusivamente a determinar la procedencia o no de la Interrupción del embarazo bajo los parámetros científicos y con apego al orden jurídico vigente". (Superintendencia de Salud. Circular No. 0003 de 2013). Destacó igualmente que se debe contar con un número adecuado de prestadores de servicios de salud habilitados para prestar dichos servicios, permitir que las mujeres accedan al mismo de manera oportuna y con calidad de servicio en todos los niveles de complejidad, que no se deben generar prácticas dilatorias en la prestación del servicio y que los departamentos, distritos y municipios deben contar con una amplia red pública, de manera que se garantice en todo el país la prestación de la IVE. En todo caso, la Superintendencia podrá adelantar un proceso e imponer una sanción a quienes se nieguen a practicar la IVE.

Presentamos dos (2) protocolos de prestación de servicios de IVE: uno utilizado en la EPS SURA y el otro en CAFESALUD EPS, los cuales contienen los parámetros expuestos por la Sentencia C-355 de 2006 de la Corte Constitucional, para, a partir de los mismos, realizar el análisis respecto a si se han tenido en cuenta las consideraciones hechas dentro del pronunciamiento de la Corte y la Circular reseñada.

\subsection{EPS SURA 2 .}

El documento proporcionado por esta EPS se titula "Guías de abordaje y seguimiento. Procedimiento para la Intervención Voluntaria del Embarazo" y contiene aspectos como: concepto de la IVE, casos en que se puede aplicar, quienes deciden sobre la continuación o no del embarazo, el tiempo establecido para la práctica del mismo, las obligaciones de las EPS e IPS en el tema, conceptos sobre la objeción de conciencia y los pasos a seguir para la solicitud de la práctica del procedimiento. 
Destacamos para el análisis, el último aspecto que consiste en:

... 1. Las usuarias afiliadas al POS de EPS SURA que soliciten la interrupción voluntaria del embarazo cuando exista grave malformación del feto, deben presentar los siguientes documentos de soporte necesarios para el estudio y generación de la orden:

Certificación expedida por médico(a) en donde se reconozca el tipo grave de malformación congénita y su incompatibilidad con la vida. En esta certificación deben constar las semanas de gestación al momento de solicitar la ILVE. Lo anterior es fundamental para definir la ruta que debe seguir la usuaria para la definición y realización del procedimiento.

Ecografía gestacional realizada en cualquier momento de la gestación previo a la solicitud de ILVE, que permita validar las semanas de gestación.

2.Las usuarias afiliadas al POS de EPS SURA que soliciten la interrupción voluntaria del embarazo cuando el embarazo constituya peligro para la vida o la salud física o mental de la mujer, deben adjuntar los siguientes documentos de soporte necesarios para el estudio y generación de la orden:

Certificación expedida por médico(a)* en caso de peligro para la vida o la salud física o certificado expedido por psicólogo* donde se reconozca el peligro que acarrea para salud mental de la mujer llevar a término el embarazo. En esta certificación deben constar las semanas de gestación al momento de solicitar la ILVE. Lo anterior es fundamental para definir la ruta que debe seguir la usuaria para la definición y realización del procedimiento.

Ecografía gestacional realizada en cualquier momento de la gestación previo a la solicitud de ILVE, que permita validar las semanas de gestación. En los casos en que la usuaria solicite ILVE aduciendo peligro para la salud mental y no se cuente con certificación expedida por Psicólogo, la IPS básica solicitará la evaluación urgente por esta especialidad a través de la Línea del Prestador. Una vez se realice la asesoría por 
parte de un psicólogo de la red convenida por EPS SURA, la institución o el profesional que expide el certificado deberá remitir a la usuaria a la plataforma en cada regional donde se generará la orden de servicio para la autorización del procedimiento y se direccionará a la paciente a la red convenida por EPS SURA.

3.Las usuarias afiliadas al POS de EPS SURA que soliciten la interrupción voluntaria del embarazo por acceso carnal o acto sexual sin consentimiento, abusivo o de inseminación artificial o transferencia de óvulo fecundado no consentidas, o de incesto, deberán adjuntar la copia de denuncia ante las autoridades competentes en la cual se especifique el hecho, así como una ecografía gestacional realizada en cualquier momento de la gestación previo a la solicitud de ILVE, que permita validar las semanas de gestación.

Para la atención de las posibles usuarias, el protocolo comienza en la IPS de profesionales de la red prestadora de servicios, con la identificación del caso, esto es, establecer en cuál de las tres (3) causales está inmersa la paciente; se continúa con la asesoría pre-IVE, consistente en hacer la respectiva anotación en la historia clínica, la usuaria decide si se realiza o no el procedimiento, se inserta nota completa en la historia, se solicita la documentación requerida para el caso; si aplica, se dirige la paciente a la plataforma regional con los documentos y con una ecografía obstétrica realizada en cualquier momento de la gestación. Ante la Regional, se genera orden de autorización para procedimiento en la institución que se defina según las semanas de gestación y se informa a la usuaria. Ante la IPS respectiva, la paciente diligencia el consentimiento informado, se practica la IVE y se continúa con la asesoría post-IVE, direccionando a la IPS básica. (Puede ingresar a planes de planificación familiar, consulta psicológica y /o psiquiátrica.

Al respecto, se concluye que esta EPS cuenta con el modelo de atención básica sugerido por el protocolo para el sector salud, sobre prevención del aborto inseguro en Colombia propuesto por el Ministerio de Salud.

\subsection{CAFESALUD EPS}

Este protocolo contiene conceptos sobre la objeción de conciencia, el procedimiento a seguir, incluyendo la atención integral para la IVE, 
advirtiendo sobre la obligación de guardar el secreto profesional, la confidencialidad del diagnóstico y su evolución. Igualmente contiene aspectos sobre la forma de realizar el feticidio teniendo en cuenta las semanas de gestación y además, las dos técnicas para la interrupción del embarazo; esto es, método quirúrgico, o con medicamentos.

Destaca que previo a realizar cualquier tratamiento de este tipo la paciente debe pasar por una consulta médica previa para revisar antecedentes patológicos, médicos, quirúrgicos, entre otros, para establecer posibles riesgos, además de determinar la edad gestacional y anatomía de genitales a través de ecografía pélvica y finalmente se realiza un examen físico para descartar enfermedades anatómicas uterinas.

Lo anterior con el fin de confirmar el embarazo, determinar la edad y localización del mismo, clasificar el riesgo biológico, psicológico y social, y asesorar a la mujer para la toma de decisiones.

Evacuada esta etapa se procede a diligenciar el consentimiento informado de IVE y del procedimiento a realizar. Acto seguido se selecciona la técnica para la terminación del embarazo (ya sea a través de medicamentos o de manera quirúrgica), y se da cita para la realización del procedimiento.

La respuesta a la solicitud se realizará en los cinco días calendarios siguientes a la misma anexando la historia clínica. Importa resaltar que la mujer puede elegir libremente entre el tratamiento quirúrgico o farmacológico, dependiendo de la edad gestacional.

Practicada la IVE se hará seguimiento y control posterior a través de consultas en donde se presta asesoría a la paciente sobre regulación de la fecundidad, asesoría en métodos anticonceptivos a través de recomendaciones realizada por el médico general, enfermera, y/o gineco-obstetra.

Es importante señalar que en la mayoría de los casos las mujeres no acuden a las instituciones prestadoras del servicio de salud, con el fin de obtener información acerca del derecho que tienen a interrumpir de manera voluntaria su embarazo, pues en el momento de indagar en estas EPS fue común la observación de que el método del cual ha sido objeto este articulo poco se realiza, lo que nos lleva a concluir que son muchos 
los embarazos que se practican de manera ilegal en el país, no obstante la despenalización del aborto que la Corte Constitucional defendió en las tantas veces referida Sentencia C-355 de 2006.

\section{CONCLUSIONES}

Todas las mujeres que deseen practicar la IVE, siempre y cuando estén bajo las tres (3) circunstancias en que la Corte Constitucional ha despenalizado el aborto, tienen derecho a acceder a los servicios que implican dicho procedimiento, contando con la información suficiente, tanto previa al procedimiento, como después de realizado, pues se debe hacer un seguimiento médico tanto físico como psicológico por los efectos que puede producir en la salud mental una decisión de interrumpir el embarazo.

Las mujeres tienen derecho a la confidencialidad y los médicos, por su parte, a guardar el secreto profesional, respetando la intimidad y dignidad de aquellas, pues es una elección a la que tienen derecho, sin realizar juicios de valor o apreciaciones morales que perjudiquen su imagen o deterioren su calidad de vida.

Ninguna entidad prestadora de servicios de salud puede negarse a la IVE, cuando la mujer se encuentre dentro de los tres (3) supuestos contenidos en la Sentencia C-355 de 2006, sin importar su condición social, económica, raza, edad, religión, etc.

En cuanto a la objeción de conciencia que puedan presentar los profesionales médicos, la misma debe ser individual y solo aplica para quien deba realizar el procedimiento y en el evento de presentarse dicha situación, las EPS e IPS deben contar con personal profesional que no tenga impedimento de esta clase para realizar la IVE.

Ni la Superintendencia de Salud, ni el Ministerio, ni la reglamentación colombiana han tocado el tema de responsabilidad administrativa de las instituciones prestadoras de servicios de salud que pueda derivarse por la demora en la práctica de la IVE a las mujeres que la solicitan, luego es importante que se legisle sobre el tema, para obligar a estas instituciones a cumplir con las directrices propuestas por los organismos reguladores, estableciendo un régimen sancionatorio, ya sea a través de multas pecuniarias o inclusive con castigos drásticos como la suspensión de la prestación del servicio de salud de las IPS que dilaten o se nieguen de manera reiterada a practicar la IVE. 


\section{REFERENCIAS BIBLIOGRÁFICAS}

HENAO, Iván Carlos. (2003) El Daño. Editorial Universidad Externado de Colombia. Bogotá.

GARAY, Oscar Ernesto. (2009). Tratado de Responsabilidad Civil en las Especialidades Médicas. Tomo I. Editorial Errepar. Buenos Aires.

MERCHÁN PRICE, Jorge. (2012) Derecho a la Vida. Ética Médica. Abusos y Atropellos. Ediciones la U. Bogotá.

GIL BOTERO, Enrique. (2013) Responsabilidad Extracontractual del Estado". Editorial Temis S.A. Bogotá.

GIL BOTERO, Enrique. (2013). Tesauro de la Responsabilidad Extracontractual del Estado. Tomo I. Editorial Temis. Bogotá.

TAMAYO JARAMILLO, Javier. (2010). Tratado de Responsabilidad Civil. Tomo I. Editorial Legis S.A. Bogotá.

COLOMBIA. ASAMBLEA GENERAL CONSTITUYENTE, Constitución Política de Colombia 1991. (04 julio, 1991). Gaceta constitucional No. 127 del 10 de octubre de 1991.

COLOMBIA. CORTE CONSTITUCIONAL DE COLOMBIA. MP, Jaime Araujo Rentería y Clara Inés Vargas Hernández. Sentencia C-355 de 2006. (10 mayo, 2006). Relatoría Corte Constitucional de Colombia, 2006.

COLOMBIA. CORTE CONSTITUCIONAL DE COLOMBIA. MP, HUMBERTO ANTONIO SIERRA PORTO. Sentencia T-841 de 2011. (3 de noviembre de 2011). Relatoría Corte Constitucional de Colombia, 2011.

BERMÚDEZ MUÑOZ, Martín. (1997). La prueba de la responsabilidad estatal derivada de la prestación de servicios médicos. Revista del Instituto Colombiano de Derecho Procesal. Vol. 2, núms. 21 y 22, 1997. MINISTERIO DE EDUCACIÓN. Recuperado en: http:// Www.colombiaaprende.edu.co/html/productos/1685/articles172254_recurso_1.pdf 
Colombia Aprende, Salud Sexual y Reproductiva. Recuperado de http://www.colombiaaprende.edu.co/html/productos/1685/articles-2254_recurso_1.pdf

MINISTERIO DE SALUD. Recuperado en https://www. minsalud.gov.co/sites/rid/Lists/BibliotecaDigital/RIDE/VS/PP/SMProtocolo-IVE-ajustado-.pdf 